\title{
Comparison of the recording dynamics of phenanthrenequinone-doped poly(methyl methacrylate) materials
}

\author{
Jose Mumbru ${ }^{\mathrm{a}, *}$, Iouri Solomatine ${ }^{\mathrm{a}}$, Demetri Psaltis ${ }^{\mathrm{a}}$, Shiuan Huei Lin ${ }^{\mathrm{b}}$, \\ Ken Y. Hsu ${ }^{c}$, Wei-Zheng Chen ${ }^{\mathrm{c}}$, Wha Tzong Whang ${ }^{\mathrm{c}}$ \\ a Department of Electrical Engineering, California Institute of Technology, Pasadena, CA 91125, USA \\ b Department of Electrophysics, National Chiao Tung University, Hsin-Chu 30050, Taiwan, ROC \\ ${ }^{\mathrm{c}}$ Institute of Electro-Optical Engineering, National Chiao Tung University, Hsin-Chu 30050, Taiwan, ROC
}

Received 8 March 2001; received in revised form 3 May 2001; accepted 10 May 2001

\begin{abstract}
A comparative analysis of phenanthrenequinone-doped poly(methyl methacrylate) materials fabricated at California Institute of Technology and National Chiao Tung University is performed in order to understand the differences exhibited in their recording and baking dynamics. (c) 2001 Published by Elsevier Science B.V.
\end{abstract}

Keywords: Holography; Photopolymer material; PQ-PMMA; Grating dynamics; Temperature effect

Phenanthrenequinone- (PQ-) doped poly(methyl methacrylate) (PMMA) [1,2] has been used as a recording material in optical memories and other holographic systems [3-6]. This material consists of a polymeric basis doped with chromophores, the PQ molecules. This material is lightweight and durable, and does not suffer from shrinkage. High optical quality samples of different shapes and thicknesses can be obtained. These properties make it an excellent candidate for holographic memory modules. In this paper, we compare the PQ-PMMA samples that we use at California Institute of Technology (Caltech) with those fab-

\footnotetext{
${ }^{*}$ Corresponding author. Fax: +1-626-568-89437.

E-mail address: jmumbru@sunoptics.caltech.edu (J. Mumbru).
}

ricated at National Chiao Tung University (NCTU) and try to understand the differences in behavior they exhibit.

Sample preparation consists of dissolving PQ molecules $(\leqslant 0.7 \%)$ in liquid methyl methacrylate (MMA) together with azo-bis-isobutyrolnitrile, a polymerization thermal initiator. This solution is poured into molds and allowed to polymerize in a pressure chamber. The preparation process followed at Caltech differs from the one followed at NCTU in the temperature at which the pressure chamber is set during polymerization. For the Caltech material, the temperature of the chamber is set to $80^{\circ} \mathrm{C}$. On the other hand, at NCTU the polymerization process is split into two steps [4,7]. First, the solution is let to rest at room temperature for approximately $120 \mathrm{~h}$ until the solution turns homogeneously viscid. At this point, the 
temperature of the chamber is increased to $45^{\circ} \mathrm{C}$ for $24 \mathrm{~h}$ to complete the process. After the polymerization is completed, the residual concentration of monomer in the Caltech samples $(\sim 1 \%)$ is much smaller than in those from NCTU $(\sim 10 \%)$. This excess of monomer will be, as we will argue, responsible for the very distinct properties of both PQ-PMMA materials.

Persistent holographic recording is achieved in PQ-PMMA by interfering two beams of coherent light inside the material. The interference pattern produces the modulation of the index of refraction of the material by optically activating the PQ molecules, in the bright fringes, and inducing their attachment to the PMMA matrix. Such attachment results in local changes of the index of refraction in the material with respect to those regions, the dark fringes, where the PQ molecules remain unattached. Recording occurs at room temperature, at which the diffusion of PQ molecules is negligible [1,8]. Therefore, two out-ofphase gratings, one formed by attached PQ and another by unattached molecules, result in a weak hologram that can be enhanced by raising the temperature, for example to $55^{\circ} \mathrm{C}$ in our experiments. At higher temperatures free PQ can diffuse, thus the grating formed by unattached molecules is erased while the one formed by the attached PQ remains unaffected, amplifying the diffraction efficiency of the hologram.

The material developed at NCTU seems to contradict the above described mechanism of hologram formation and amplification, since high diffraction efficiency holograms have been reported in PQ-PMMA samples with recording at room temperature without the need for any postrecording thermal treatment of the samples [5,7]. It is believed that in this case, the higher concentration of monomer in the sample plays a key role in the recording process, because the PQ molecules seem to prefer to attach to MMA rather than to the PMMA matrix [7]. An alternative explanation for hologram formation is then regarded, in which at room temperature MMA and PQ molecules can diffuse in the material. However, the PQ-MMA groups, formed due to photoexcitation of the PQ during recording, experience only minimal diffusion. Thus no baking is required to amplify the hologram, since continuous diffusion of $\mathrm{PQ}$ is building up the PQ-MMA grating while at the same time erasing any out-of-phase grating of free PQ.

In order to find a common theory that can satisfactorily explain the distinct hologram dynamics exhibited by the two PQ-PMMA materials, comparative experiments to monitor the recording and baking processes have been performed at Caltech on samples of both universities. The samples used in the comparison are a $1 \mathrm{~mm}$ thick disk of $40 \mathrm{~mm}$ in diameter made at Caltech and a bar $20 \mathrm{~mm} \times 36 \mathrm{~mm}$ in cross-section and 2.4 $\mathrm{mm}$ thick made at NCTU. The concentration of PQ is $0.5 \mathrm{wt} . \%$, the same in both samples.

To study the recording dynamics, a hologram is written using the $514 \mathrm{~nm}$ line of an argon laser in a symmetric transmission geometry setup. The angle of incidence of each one of the recording beams is approximately $30^{\circ}$ with respect to the surface normal of the sample, resulting in a grating period $\Lambda$ of $0.5 \mu \mathrm{m}$. A $633 \mathrm{~nm} \mathrm{He}-\mathrm{Ne}$ laser beam, outside the absorption band of PQ-PMMA, is Braggmatched to the hologram to study the post-exposure dynamics of the sample once the $514 \mathrm{~nm}$ beams have been shut off.

Using the above-described samples, a weak hologram is recorded in the NCTU and Caltech samples. The exposure energies are 0.3 and $0.5 \mathrm{~J} /$ $\mathrm{cm}^{2}$ respectively. This energy is delivered to the sample in an interval of less than $10 \mathrm{~s}$, minimizing thus the effect of instability in the recording setup. Fig. 1 shows the dark enhancement of the diffraction efficiency of the hologram in the NCTU (solid line) and Caltech (dashed line) samples during a period of $5 \mathrm{~h}$ after recording. The square root of each curve, the grating strength, has been fitted to a mono-exponential function with time constant $23.05 \mathrm{~min}$ for the NCTU sample and $16.96 \mathrm{~min}$ for the Caltech sample. From these results, there are two issues that need to be interpreted: First of all, the reason why this time constant seems to be the same in both cases. And secondly, why the saturation level in the NCTU sample occurs at a much higher value of diffraction efficiency than in the Caltech sample.

As far as the time constant is concerned, the experimental results on both PQ-PMMA media 


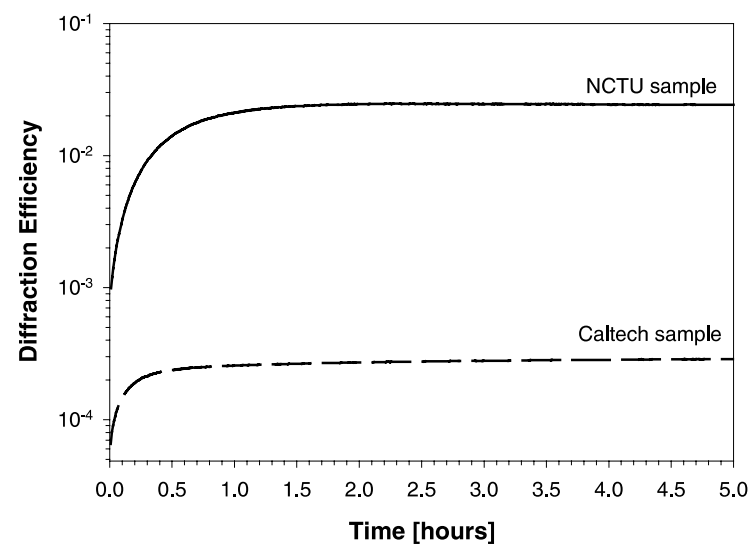

Fig. 1. Comparison between the evolution of the diffraction efficiency of a hologram recorded in the NCTU sample (-) and in the Caltech sample (-- ) for $5 \mathrm{~h}$ after exposure at room temperature.

suggest that the same mechanism is taking place in both samples and dominates the post-recording dynamics. Then the question is to identify what this process is. It is central to the understanding of the dynamics of the material to verify the possibility that free PQ molecules may diffuse in the polymeric matrix at room temperature, at which the experiments were performed. This hypothesis can be checked by solving the one-dimensional case of the diffusion equation as described in earlier papers by Veniaminov et al. [1,8]. The diffusion constant of the free PQ molecules, $D_{1}$, relates to the measured time constant, $\tau$, as:

$\frac{4 \pi^{2}}{\Lambda^{2}} D_{1}=\frac{1}{\tau}$

Taking $\Lambda=0.5 \mu \mathrm{m}$ and $\tau \approx 20 \mathrm{~min}$, the diffusion constant would be $10^{-11} \mathrm{~m}^{2} / \mathrm{s}$ at $25^{\circ} \mathrm{C}$. This value is far from the value of $10^{-21} \mathrm{~m}^{2} / \mathrm{s}$ reported by Veniaminov and Sedunov [1], even if the latter is adjusted to account for the difference of plasticity of the two samples. Therefore we can conclude that the observed dynamics does not correspond to the diffusion of free PQ molecules. In fact, we believe that the enhancement of the hologram after recording is due to the attachment of photoactivated PQ radicals to either MMA or PMMA. Consequently, the observed dynamics is the rate at which this attachment is taking place. This process is much faster than the diffusion of PQ molecules and, in the experiment, it is completed within the first two hours after exposure.

Regarding the question why the diffraction efficiency at its saturation value in the NCTU sample is much higher than it is in the Caltech sample, in the experiment shown in Fig. 1 it is about two orders of magnitude, and having already ruled out the possibility of PQ molecules diffusing at $25^{\circ} \mathrm{C}$ from dark into bright regions of the material, the only species that could still diffuse would be the MMA. The fact that MMA is about two and a half times lighter than PQ can explain an increase of its diffusion constant in one or two orders of magnitude, however this would still insufficient to account for the value obtained from the experiment.

Conversely, we believe that the origin of such a difference in diffraction efficiency is partly found in the ratio of the polarizabilities of the PQ molecule in its different states [1,8]. If $\alpha_{1}, \alpha_{2}$ and $\alpha_{3}$, are the polarizabilities and $C_{1}(x, t), C_{2}(x, t)$ and $C_{3}(x, t)$ are the concentrations of free PQ, PQ attached to PMMA, and PQ attached to MMA respectively, then upon recording the two offsetting gratings are in the case of the Caltech PQ-PMMA:

$\Delta n(x, t)=\alpha_{2} C_{2}(x, t)-\alpha_{1} C_{1}(x, t)$

where the minus sign indicates the $\pi$ phase shift between the gratings, and for the NCTU PQPMMA:

$\Delta n(x, t)=\alpha_{3} C_{3}(x, t)-\alpha_{1} C_{1}(x, t)$

It is then conjectured that the ratio between $\alpha_{3}$ and $\alpha_{1}$ must be larger than the ratio between $\alpha_{2}$ and $\alpha_{1}$ resulting in a stronger hologram. It is unlikely though that this higher contrast obtained when PQ attaches to MMA can totally account for the different behavior of the two samples. Furthermore, if it is taken into account the fact that the number of PQ molecules $(0.5 \mathrm{wt} . \%)$ is in either case much lower than the number of molecules of monomer (1 wt.\% in the Caltech and $10 \mathrm{wt} . \%$ in the NCTU samples), it is not unreasonable then to think that even in the Caltech PQ-PMMA part of the grating is recorded in PQ molecules attaching to the monomer. 
What we consider to be the main reason for the discrepancy in behavior of the samples can be found in a paper by Popov et al. [6]. As described in the paper, PQ can act as photoinitiator of polymerization in those situations where there is an important excess of residual monomer, as it happens in the NCTU material. In these situations, the photopolymerization is combined, and can even dominate, the diffusion mechanism of hologram formation. In other words, in the NCTU PQ-PMMA, the photoactivated PQ molecules act as initiators of the polymerization before they eventually attach to MMA, resulting in substantial modulation of the index of refraction, and thus strong holograms.

The effect of baking the samples on the diffraction efficiency of the holograms has also been investigated. Six holograms were recorded on different locations on the Caltech sample with exposure energies ranging from 2 to $20 \mathrm{~J} / \mathrm{cm}^{2}$. The recording sensitivity of the material can be obtained by fitting the grating strength of the holograms to a line (Fig. 2(a)). In the figure, only five of the six holograms are presented. The hologram with exposure energy of $20 \mathrm{~J} / \mathrm{cm}^{2}$ is omitted because of saturation of the material. From the measurements, a pre-baking sensitivity of 0.103 $\mathrm{cm} / \mathrm{J}$ is obtained. The sample was then taken out of the recording setup and placed in an oven at $55^{\circ} \mathrm{C}$. The diffraction efficiency of the holograms has been monitored and it is plotted in Fig. 3(a) as a function of the baking time. As expected, at $55^{\circ} \mathrm{C}$ the diffusion of free PQ molecules is greatly enhanced so after just $18 \mathrm{~h}$ in the oven, the free PQ grating has been practically totally erased. On average, the hologram amplified their diffraction efficiency by a factor of 19.62 , achieving values as high as $60 \%$ for the longest exposures. At this point, the "after baking" sensitivity is measured, resulting in $0.396 \mathrm{~cm} / \mathrm{J}$ (Fig. 2(a)). This increase is consistent with the amplification of the holograms during baking.

A similar experiment is performed on the NCTU sample. Five holograms were recorded with energetic exposures from 0.25 to $1.5 \mathrm{~J} / \mathrm{cm}^{2}$. The holograms have been allowed to develop for $2.5 \mathrm{~h}$ in the dark before placing the sample in the oven. At this point the holograms were measured, (a)

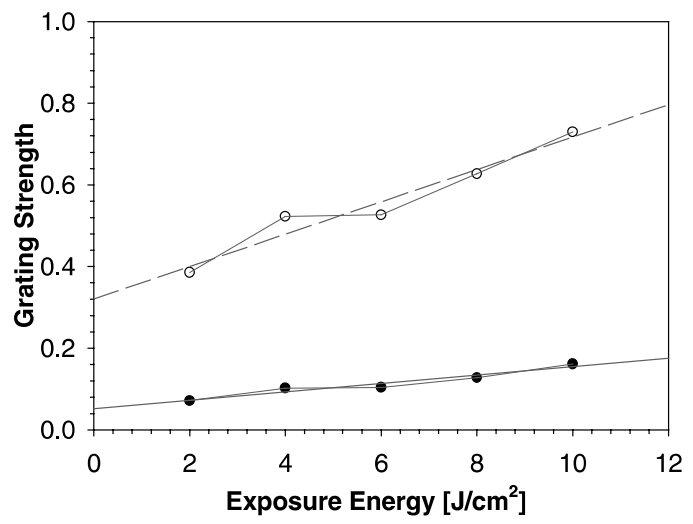

(b)

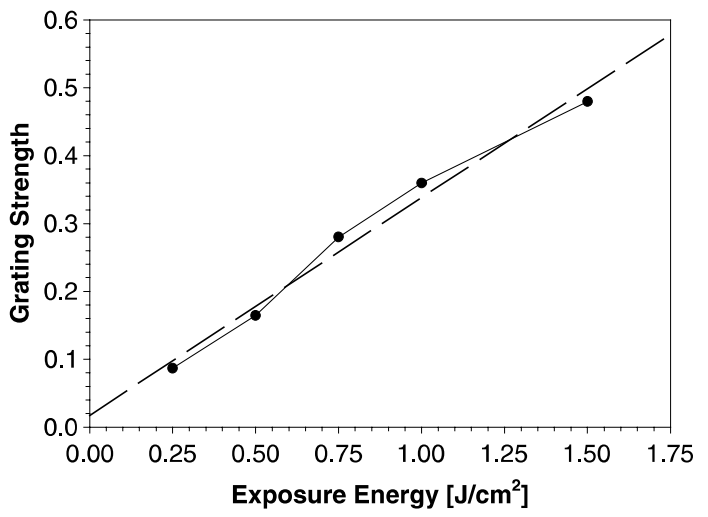

Fig. 2. Comparison of the recording sensitivity: (a) Caltech sample: steady-state grating strength of the holograms before baking (black dots and solid line) and after $18 \mathrm{~h}$ baking (white dots and dashed line) as a function of the exposure energy; (b) NCTU sample: steady-state grating strength of the holograms (before baking only).

and their grating strengths fitted to a line (Fig. 2(b)), resulting in an equivalent sensitivity of 1.34 $\mathrm{cm} / \mathrm{J}$, which is approximately 3.4 times higher than the one obtained in the Caltech sample. The diffraction efficiency of the holograms was monitored after incremental baking was being applied to the sample. The result that was obtained, as shown in Fig. 3(b), is clearly different from the one experienced by the Caltech sample. The holograms are not enhanced but degraded by the baking. The evolution of the strength of the gratings can be fitted to exponential functions, and the time constant is estimated to be $9.65 \mathrm{~h}$.

The reason for the degradation is that at $55^{\circ} \mathrm{C}$ both free PQ molecules and PQ-MMA groups can diffuse in the polymer matrix, so it is not that one 
(a)

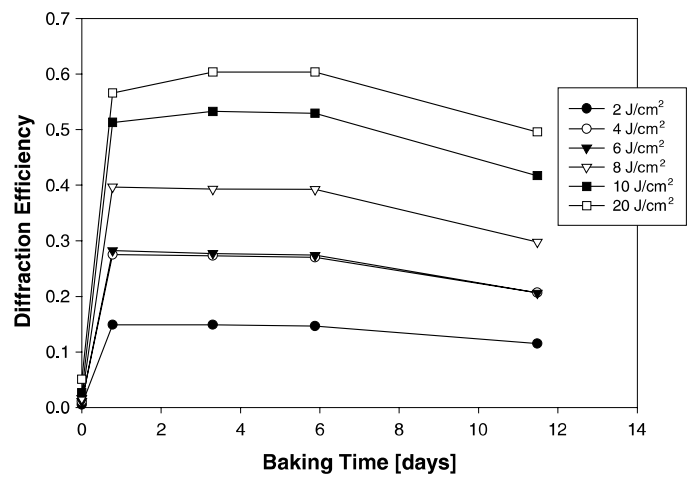

(b)

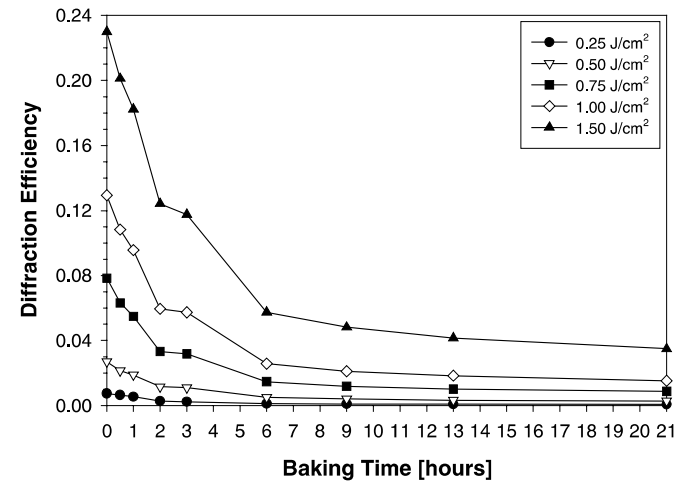

Fig. 3. Effect of baking (at $55^{\circ} \mathrm{C}$ ) on the diffraction efficiency as a function of time: (a) amplification of the holograms recorded in the Caltech sample; (b) erasure of the holograms recorded in the NCTU sample.

of the two out of phase gratings is being erased but both of them resulting in the destruction of the hologram. Once again, the estimated time constant can be converted to a diffusion constant using Eq. (1). For $\Lambda=0.5 \mu \mathrm{m}$ and $\tau=9.65 \mathrm{~h}$, the equivalent diffusion constant for free PQ and PQ-MMA would be $1.9 \times 10^{-19} \mathrm{~m}^{2} / \mathrm{s}$ at $55^{\circ} \mathrm{C}$, which is in good agreement with the value of $1.6 \times 10^{-19} \mathrm{~m}^{2} / \mathrm{s}$ extrapolated from data reported by Veniaminov et al. [1].

The effect of prolonged baking of the sample has also been analyzed. In the case of the Caltech sample, the strength of two holograms, each with exposure energy of $20 \mathrm{~J} / \mathrm{cm}^{2}$, has been monitored for up to 44 days of continuous baking (Fig. 4(a)). After the initial amplification, further baking did not significantly degrade the grating formed by the PQ attached to the PMMA. On the other hand, in the NCTU material the destruction of the holo- (a)

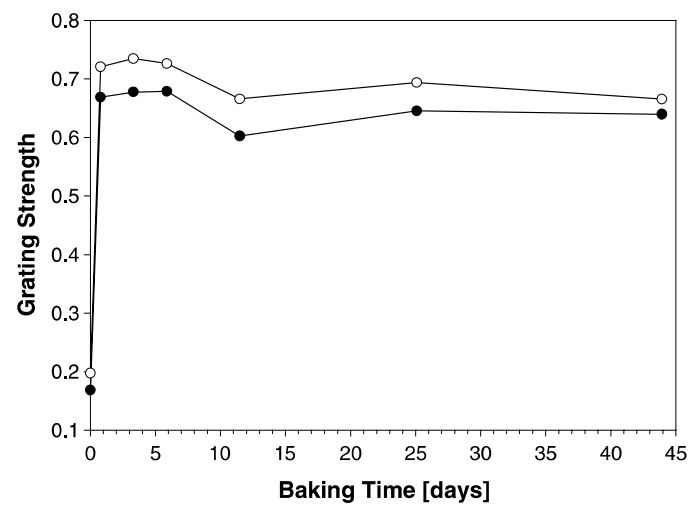

(b)

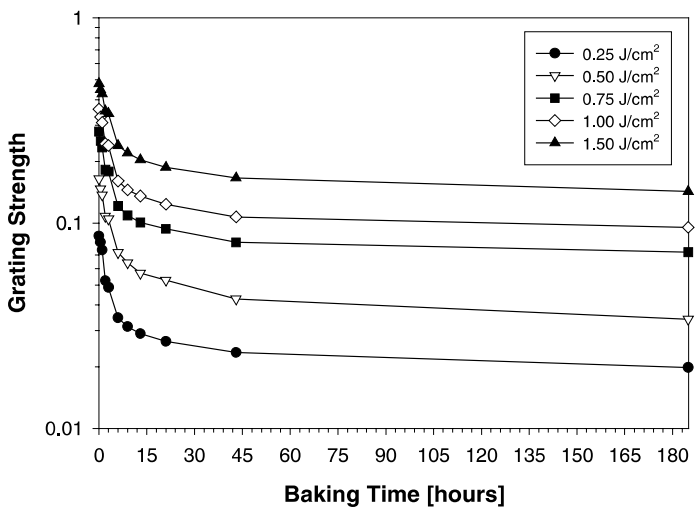

Fig. 4. Effect of prolonged baking: (a) evolution of the grating strength of two holograms (exposure energy $20 \mathrm{~J} / \mathrm{cm}^{2}$ ) recorded in the Caltech sample as a function of baking time; (b) decay of the grating strength of the holograms recorded in the NCTU sample as a function of baking time.

gram is not complete. As it can be seen in Fig. 4(b), the grating strength curves initially decay due to the mentioned diffusion process, but eventually they flatten off to some final value. The remaining grating is due to the modulation of index refraction caused by the polymerization of monomer in the illuminated areas, as well as by the fraction of PQ that attached to the PMMA. This grating is stable and will not degrade with additional baking. The presence of a residual grating supports the hypothesis that the polymerization mechanism is responsible for the hologram recording in the NCTU material.

We conclude from the comparison between the NCTU and Caltech PQ-PMMA material that the difference in their behavior lies in the different concentration of residual MMA in the samples. 
Experimental evidence shows that during recording PQ molecules attach to MMA but no diffusion takes place at room temperature. However, the excess of monomer during recording enables photoinduced polymerization as a mechanism for hologram formation leading to high diffraction efficiencies without the need of baking. The grating formed by the PQ-MMA groups is unstable and it can be erased within a few hours of baking.

\section{Acknowledgements}

The authors want to acknowledge Andrei V. Veniaminov and Gregory J. Steckman for helpful discussion. This research is funded by DARPA (grant no. F30601-98-1-0199) and by the National Science Foundation Engineering Research Center grant to Caltech.

\section{References}

[1] A.V. Veniaminov, Yu.N. Sedunov, Polym. Sci. A 38 (1996) 59.

[2] V.I. Sukhanov, J. Opt. Technol. 61 (1994) 49.

[3] J.E. Ludman, J.R. Riccobono, N.O. Reinhand, I.V. Semenova, Y.L. Korzinin, S.M. Shahriar, H.J. Caulfield, J.M. Fournier, P. Hemmer, Opt. Eng. 36 (6) (1997) 17001705.

[4] G.J. Steckman, I. Solomatine, G. Zhou, D. Psaltis, Opt. Lett. 23 (16) (1998) 1310-1312.

[5] K.Y. Hsu, S.-H. Lin, W.T. Whang, W.Z. Chen, Holographic data storage using photopolymers, in: F.T. Yu, S. Yin (Eds.), Proc. SPIE "Photorefractive Fiber and Crystal Devices: Materials, Optical Properties, and Applications V", vol. 3801, 1999, pp. 66-74.

[6] A. Popov, I. Novikov, K. Lapushka, I. Zyuzin, Yu. Ponosov, Yu. Ashcheulov, A. Veniaminov, J. Opt. A: Pure Appl. Opt. 2 (5) (2000) 494-499.

[7] S.H. Lin, K.Y. Hsu, W.-Z. Chen, W.T. Whang, Opt. Lett. 25 (7) (2000) 451-453.

[8] A.V. Veniaminov, V.F. Goncharov, A.P. Popov, Opt. Spectrosc. 70 (1991) 505. 